\title{
The detectability of fast gamma-ray blazar flares from magnetic reconnection with the Fermi Large Area Telescope
}

\author{
M. Petropoulou, ${ }^{a, *}$ M. Meyer, ${ }^{b}$ I. Christie ${ }^{c}$, on behalf of the Fermi-LAT collaboration \\ ${ }^{a}$ Department of Physics, National \& Kapodistrian University of Athens, University Campus, Zografos, \\ 15784, Greece \\ ${ }^{b}$ Erlangen Centre for Astroparticle Physics, University of Erlangen-Nuremberg, Erwin-Rommel-Str. 1, \\ D-91058 Erlangen, Germany \\ ${ }^{c}$ Northrop Grumman \\ E-mail: mpetropo@phys.uoa.gr
}

The physical mechanism for the production of fast gamma-ray variability in blazars remains debated. Plasmoids - magnetized quasi-circular structures of plasma formed self-consistently in reconnecting current sheets - are ideal candidates for the production of broadband variable non-thermal emission. Using state-of-the-art kinetic simulations of magnetic reconnection and radiative transfer calculations, we generate artificial gamma-ray light curves that would be observed with the Fermi Large Area Telescope (LAT). Our goal is to investigate if characteristic features of the theoretical light curves, such as the ultra-rapid gamma-ray flares predicted by the reconnection model, are detectable with the typical Fermi-LAT observations. A comparison with observed luminous and fast gamma-ray flares from flat spectrum radio quasars (FSRQs) reveals that magnetic reconnection events lead to comparable flux levels and variability patterns, especially when the reconnection layer is slightly misaligned with the line of sight. Emission from fast plasmoids moving close to the line of sight could explain fast variability on the time scales of minutes for which evidence has been found in observations of FSRQs. Our results motivate improvements in the existing reconnection model for blazars as well as dedicated searches for fast variability in LAT data as evidence for magnetic reconnection events.

$37^{\text {th }}$ International Cosmic Ray Conference (ICRC 2021)

July 12 th - 23rd, 2021

Online - Berlin, Germany

\footnotetext{
*Presenter
} 


\section{Introduction}

Blazars are the most powerful persistent astrophysical sources of non-thermal electromagnetic radiation in the Universe, with spectral energy distributions (SEDs) spanning $\sim 15$ decades in energy, from radio frequencies up to high-energy $\gamma$-rays. Blazars are also characterized by flux variability that is frequency-dependent and manifests in a variety of timescales, ranging from minutes to years [for a review, see 1].

The brightest $\gamma$-ray flares of six flat spectrum radio quasars ${ }^{1}$ (FSRQs) within $\sim 10 \mathrm{yr}$ of observations with the Fermi Large Area Telescope (LAT) ${ }^{2}$ have been recently studied by [5]. Evidence for sub-hour timescales (as short as minutes) has been presented for two FSRQs in the sample (3C 279 and CTA 102) at a $\sim 2 \sigma$ post-trial significance. For two additional sources (PKS 1510-089 and 3C 454.3) decay times on timescales similar or less than the satellite's orbit (95 minutes) were found [see Fig. 6 in Ref. 5].

Minute-scale $\gamma$-ray flares are of particular importance as they are a manifestation of the underlying physical processes that power the multi-timescale temporal variability in blazars and can constrain models of $\gamma$-ray production in jets [e.g. 6-9]. Doppler factors that are much higher than those inferred from radio observations [e.g. 10,11] are usually required to explain luminous fast $\gamma$-ray flares and avoid internal attenuation of $\gamma$-rays [e.g. 12,13]. The requirements for the jet's Lorentz factor $\Gamma_{j}$ can be, however, relaxed if the emitting region moves relativistically within the jet with Lorentz factors $\Gamma_{\text {co }}$ of a few. The Doppler factor is then defined as $\delta \sim \Gamma_{\mathrm{co}} \Gamma_{j}$, which can easily exceed 40 for typical values of $\Gamma_{j}$.

Magnetic reconnection - a process where magnetic energy is transferred to the plasma bulk motion and acceleration of particles - offers a natural way of producing compact magnetized structures, the so-called plasmoids, moving at relativistic speeds with respect to the co-moving frame of the jet [e.g. 14, 15]. Plasmoids, which contain nonthermal particles, can accelerate due to magnetic stresses up to a terminal Lorentz factor $\Gamma_{\mathrm{co}} \approx 3(\sigma / 10)^{1 / 2}$, where $\sigma \gtrsim 1$ is the jet's plasma magnetization [16, 17]. In addition to bulk acceleration, plasmoids grow in size by accreting plasma either through the secondary current sheets between plasmoids or through mergers with other neighboring plasmoids. Because of their properties, plasmoids have been suggested as candidate sites for the production of short-duration $\gamma$-ray flares from blazars [18, 19].

By coupling two-dimensional (2D) particle-in-cell (PIC) simulations of relativistic reconnection with a time-dependent radiative transfer code, [20] computed the nonthermal emission from a chain of plasmoids formed in a macroscopic current sheet of half-length $L$. At any given time, an observer receives radiation from a large number of plasmoids, which are present in the layer, with different sizes and Doppler factors. Plasmoids that move with mildly relativistic speeds (in the jet frame) and have intermediate sizes (e.g. $\gtrsim 0.05 \mathrm{~L}$ ) were found to contribute significantly to the overall emission. The superposition of their emission can result in a slow varying component of the light curve, i.e., an envelope of emission, as first proposed by [18], while smaller plasmoids (with sizes $\lesssim 0.01 L$ ), which typically move with relativistic speeds (i.e. $\Gamma_{\text {co }} \approx \sqrt{\sigma}$ ), can result in

\footnotetext{
${ }^{1}$ These are traditionally differentiated from other blazar subclasses by the equivalent width of lines in their optical spectra [2], but see also [3] for a recent review.

${ }^{2}$ The LAT is a pair-conversion telescope that detects $\gamma$-rays in the energy range between $30 \mathrm{MeV}$ and beyond $300 \mathrm{GeV}$ and surveys the full sky every $\sim 3 \mathrm{hr}$ [4].
} 


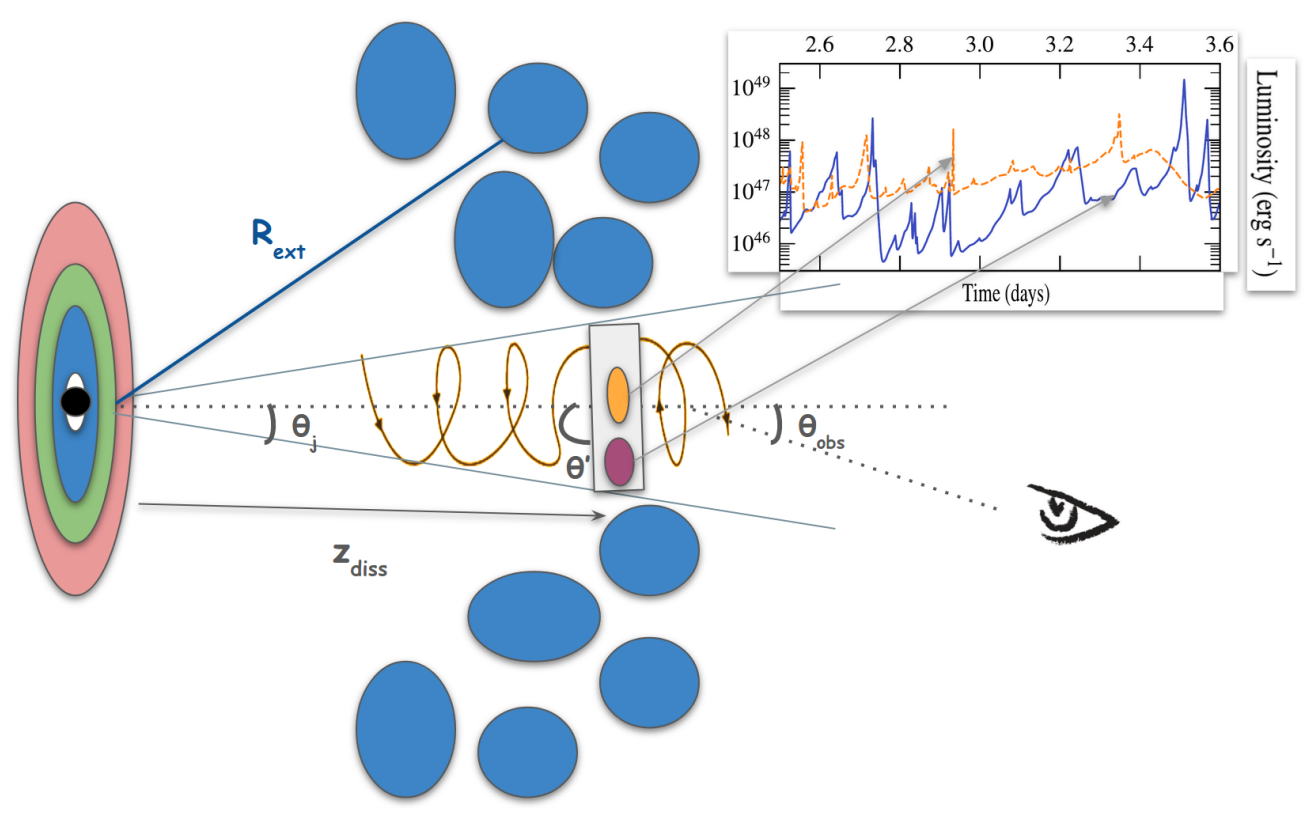

Figure 1: Schematic illustration of our model for $\gamma$-ray flaring FSRQs (not to scale). The BLR (blue colored blobs) is described by a thin spherical shell of characteristic radius $R_{\text {ext }}$ and bolometric luminosity $L_{\text {ext }}$. A current sheet of half-length $L$ forms in a conical jet of half-opening angle $\theta_{j} \approx \Gamma_{j}^{-1}$ at a distance $z_{\text {diss }} \approx \Gamma_{j} L \lesssim R_{\text {ext }}$ from an accreting supermassive black hole. The current sheet is oriented at an angle $\theta^{\prime}$ with respect to the jet axis (as measured in the jet's rest frame). Colored blobs in the current sheet indicate plasmoids that form due to the tearing instability and accelerate due to magnetic tension forces to relativistic speeds [17]. Non-thermal emission is produced by relativistic electrons that reside in plasmoids. The emission is first computed in the plasmoid rest frame and then it is mapped to the rest frame of an observer, positioned at an angle $\theta_{\text {obs }}$ with respect to the jet axis. Each plasmoid produces $\gamma$-ray transient emission, with duration and luminosity that are determined by the plasmoid size and velocity. The emission from all plasmoids produces a variable $\gamma$-ray light curve, as shown in the top right panel.

luminous and ultra-rapid flares that show up on top of the envelope emission [see Fig. 9 in Ref. 20]. A sketch of the proposed model for $\gamma$-fay blazar flares is shown in Fig. 1.

Here, we summarize our finding if certain features of the theoretical light curves predicted by the reconnection model are detectable with typical Fermi-LAT observations [21]. For this purpose, we generate artificial LAT light curves for two prototypical FSRQs, 3C 273 and 3C 279, and compare the results with actual LAT observations.

\section{Generation of artificial Fermi-LAT light curves}

First, we extend previous work of Ref. [20] by performing simulations for different orientations of the current sheet, jet bulk Lorentz factor, viewing angles, dissipation distances, and luminosities of the external photon fields. The parameters of the simulations are shown in Table 1. Other parameters of the simulation are set to the values chosen in [20].

We then use these theoretical light curves (see top panels in Fig. 2) to construct artificial Fermi-LAT light curves of two well-studied FSRQs, 3C 273 and 3C 279. The steps of our analysis are listed below: 


\begin{tabular}{lcccc} 
& \multicolumn{3}{c}{ Model } \\
& A & B & C & D \\
Bulk Lorentz factor, $\Gamma_{j}$ & 12 & 24 & 24 & 24 \\
Observer's angle, $\theta_{\mathrm{obs}}(\mathrm{deg})$ & 0 & 0.2 & 0 & 0 \\
Angle between layer and jet axis (in jet frame), $\theta^{\prime}(\mathrm{deg})$ & 0 & 0 & 30 & 0 \\
Dissipation distance, $z_{\text {diss }}(\mathrm{pc})$ & 0.2 & 0.4 & 0.4 & 0.4 \\
Bolometric luminosity of external radiation, $L_{\mathrm{ext}}\left(10^{45} \mathrm{erg} \mathrm{s}^{-1}\right)$ & 4 & 4 & 4 & 10 \\
Luminosity of two-sided jet, $L_{\mathrm{j}}\left(10^{47} \mathrm{erg} \mathrm{s}^{-1}\right)$ & 1 & 5 & 5 & 5 \\
Target blazar & 3C 273 & $3 \mathrm{C} 273$ & $3 \mathrm{C} 273$ & $3 \mathrm{C} 279$ \\
\hline
\end{tabular}

Table 1: Parameters used for the calculation of theoretical $\gamma$-ray light curves from magnetic reconnection in blazar jets.
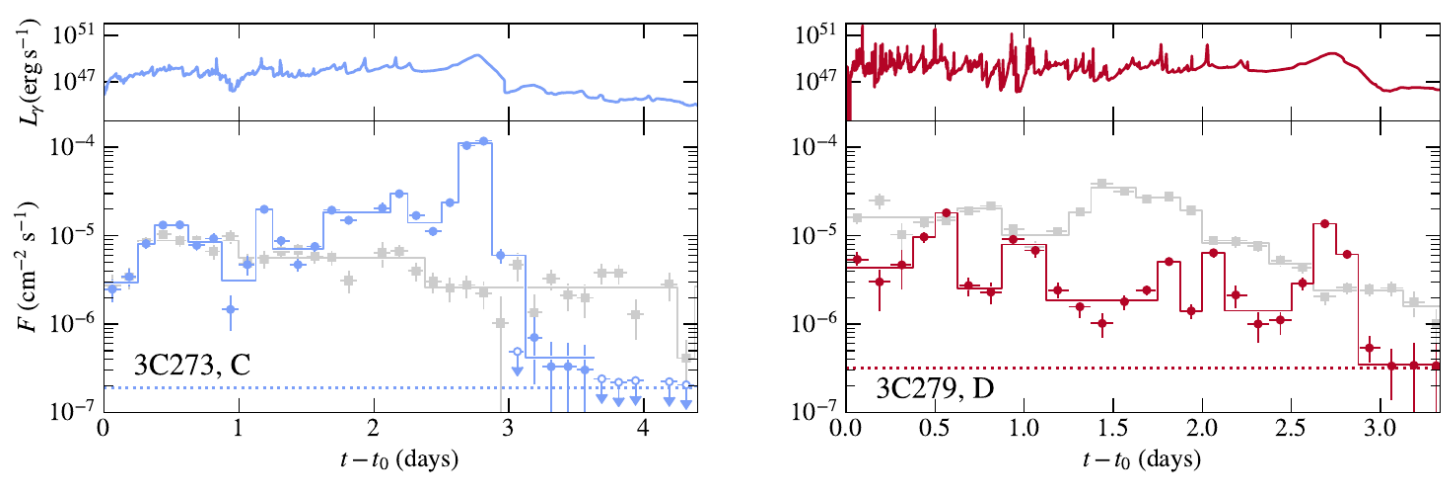

Figure 2: Theoretical (upper panels), artificial (colored symbols in lower panels), and observed (grey markers in lower panels) $\gamma$-ray light curves in the $0.1-300 \mathrm{GeV}$ energy band for models $\mathrm{C}$ and $\mathrm{D}$. A $3 \mathrm{hr}$ binning is used for the observed and artificial LAT light curves. Solid lines show the Bayesian block representation of the light curves. The light curves are plotted with respect to the start time of the observation, $t_{0}=55094.69 \mathrm{MJD}$ and 58140.66 MJD for 3C 273 and 3C 279, respectively.

1. We conduct standard LAT analysis for time periods of strong $\gamma$-ray activity. We use fermipy ${ }^{3}$ version 0.19.0 [22] and run the standard fermitools ${ }^{4}$, version 1.2.23. We extract binned light curves from the astrophysical data, shown as as grey markers in the lower panels of Fig. 2.

2. In the best-fit model of the region of interest (ROI), we replace the central FSRQ with the magnetic reconnection model assuming a power-law spectrum with photon index 2 , and taking into account attenuation by the broad line region (BLR) photons and the extragalactic background light (EBL).

3. We perform a simulation of the modified ROI and redo the LAT analysis to generate the binned artificial light curve (see colored symbols in the lower panels of Fig. 2).

We also compute the periodograms of the $3 \mathrm{hr}$-binned observed and artificial light curves, which we fit with power-law power spectral densities (PSDs), $\operatorname{PSD}(v) \propto v^{-k}$, where the frequency $v$ is

${ }^{3}$ https://fermipy . readthedocs . io

${ }^{4}$ https://fermi.gsfc.nasa.gov/ssc/data/analysis/ 

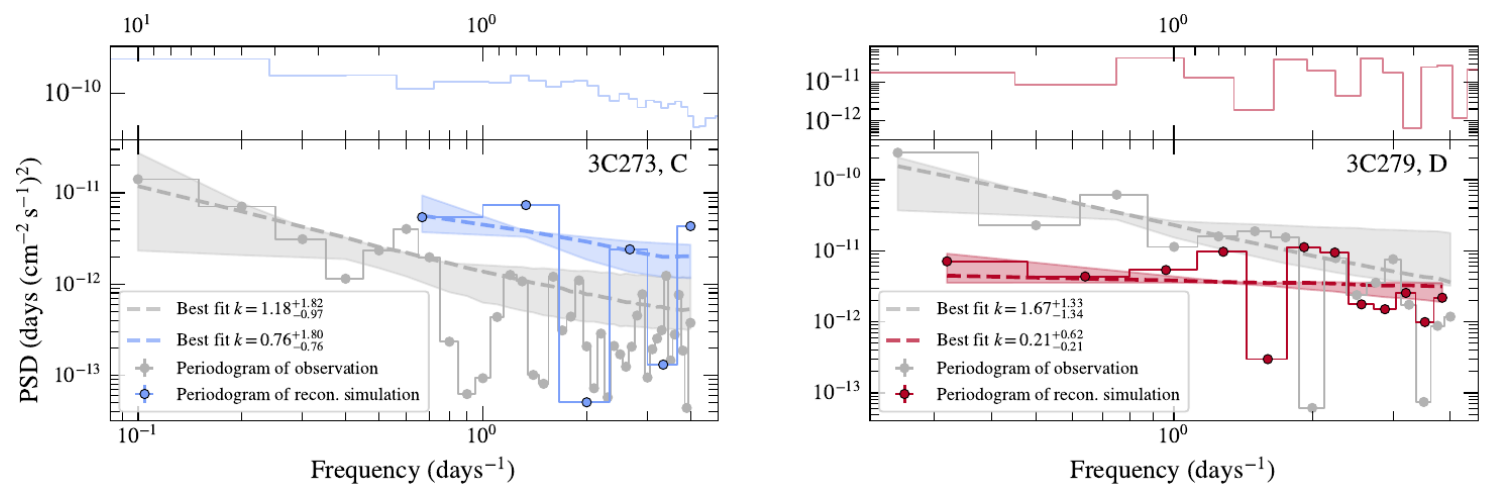

Figure 3: Periodograms of $\gamma$-ray light curves of 3C 273 and 3C 279: theoretical (upper panels), artificial (colored symbols in lower panels), and observed (grey symbols in lower panels) light curves. The best-fit values for $k_{\mathrm{obs}}$ and $k_{\mathrm{art}}$ are provided in the legend. Contours indicate the $2 \sigma$ uncerntainties.

given by the inverse of time. To fit the periodograms we follow the methodology of [5], which is based on the work of [23] and [24] (for details, see [21]). The results for 3C 273 and 3C 279 are shown in Fig. 3 for two reconnection models.

\section{Results}

\subsection{Comparison of artificial and observed light curves}

The flux of the artificial light curve in Model C is broadly consistent with the observed flux of the flare under study (see left panel in Fig. 2). On the contrary, the artificial light curve of Model D cannot match the flux of the brightest flare observed from 3C 279, even though the short duration flares of the theoretical light curve (see spikes on top right panel of Fig. 2) can reach similar levels as the observed flux.

Much of the short time variability that is visible in the theoretical light curves of both sources is lost due to the chosen binning ( $3 \mathrm{hr}$ ) and averaging. In fact, none of the sharp spikes of the theoretical light curves is individually recovered in the artificial light curves, because they all have durations less than the adopted time bin of $3 \mathrm{hr}$. As a result, most of the features seen in the artificial light curves as individual flares are in reality the superposition of multiple unresolved flares powered by individual plasmoids in the layer. Only the longest duration flare, which is produced by the single largest plasmoid in the layer, is recovered in the artificial light curves of all models (see e.g. the last Bayesian block of artificial light curves). Binning of the light curves on shorter timescales would not yield sufficient counts, thus leading to upper limits.

When looking at the timing properties of the artificial and observed light curves in the frequency domain, we find that both have PSDs that can be approximated by power laws (see Fig. 3). The best-fit values for the PSDs of artificial light curves are compatible with $k_{\text {art }}=0$ (white noise), while only $2 \sigma$ upper bounds can be derived. This is due to the short time duration of the magnetic reconnection event, which prevents us from deriving the periodograms at lower frequencies, and the $3 \mathrm{hr}$ binning applied to the light curves, which prevents the calculation of the periodogram at higher frequencies. The PSDs of the full theoretical light curves (not explicitly shown) have usually steeper slopes, ranging from $k_{\text {theo }} \sim 1.4$ (Model D) to $k_{\text {theo }} \sim 2.7$ (Model C). However, when these 

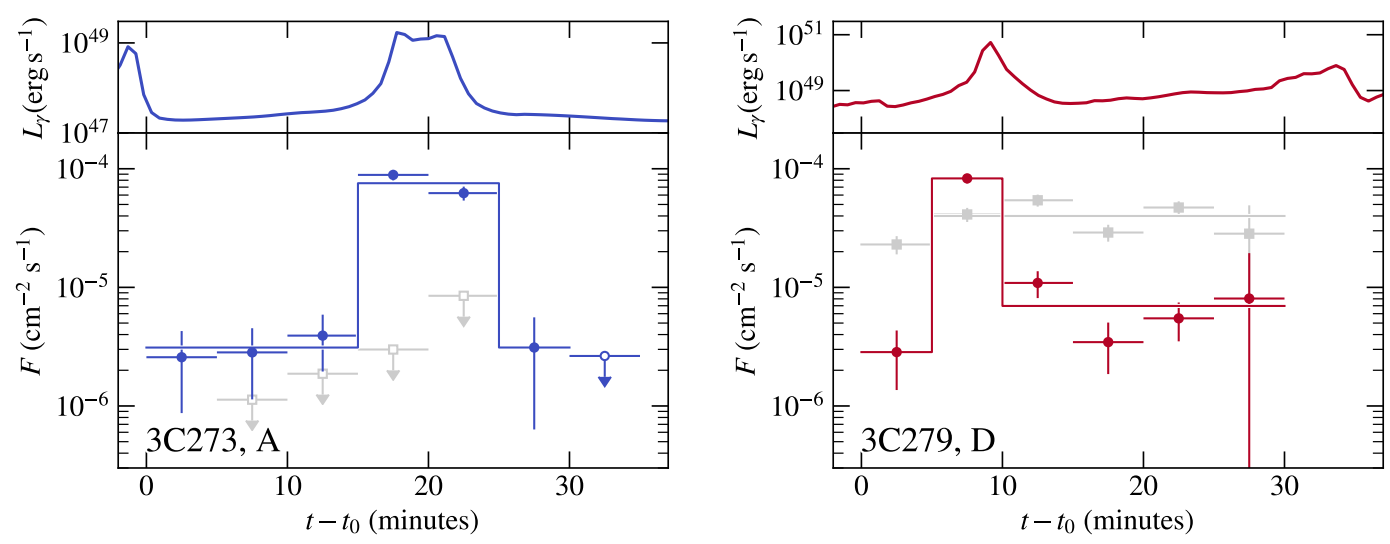

Figure 4: Theoretical (upper panels) and artificial LAT light curves with 5-minute binning (lower panels) for 3C 273 (left panel) and 3C 279 (right panel). In both panels, grey markers indicate the observed fluxes or upper limits. The theoretical light curves are shifted such that one short-duration peak of the theoretical light curve coincides with a good time interval. Here, $t_{0}=55094.69$ MJD and $t_{0}=57,189.08$ MJD for 3C 273 and 3C 279, respectively.

are limited to the frequency range of the LAT observation (see top panels of Fig. 3) we find $k_{\text {art }}<1$. The observed light curves have steeper PSDs with $k_{\mathrm{obs}} \sim 1$. These are however still compatible within statistical uncertainties.

\subsection{Minute-scale variability}

To test if even shorter variability time scales (i.e. $t_{\mathrm{var}}<3 \mathrm{hr}$ ) from reconnection can be observed, we repeat the analysis for one good time interval (GTI). This is defined as the time interval where the source is within the field of view of the satellite. Within one Fermi-LAT orbit such a GTI has usually a duration of the order of $\sim 30 \mathrm{~min}$. We shift the theoretical light curve in time so that one of the luminous and fast flares falls inside a GTI and repeat our light-curve analysis, this time using a binning of 5 min. Our results are presented in Fig. 4.

In both cases, the artificial light curves exhibit a luminous and fast flare, produced by one of the smaller and faster plasmoids in the layer. The peak flux of the flare above $100 \mathrm{MeV}$ in both models is $\sim 10^{-4} \mathrm{~s}^{-1} \mathrm{~cm}^{-2}$. Such high fluxes have never been reported for 3C 273 , while they are close to the highest flux ever reported for 3C 279 in 2015 June [13]. By tweaking the parameters of the magnetic reconnection simulation, such as the viewing angle of the layer, one can reduce the flux to the observed values and prolong the duration, thus having better agreement to the observations.

\section{Summary \& Conclusions}

We have tested the reconnection model for $\gamma$-ray blazar variability against Fermi-LAT data by taking into account the instrumental effects of the LAT (e.g. effective area and location of source in detector's field of view). We have created artificial Fermi-LAT light curves based on magnetic reconnection simulations for parameters relevant to FSRQs. We showed that the artificial light curves are in general able to reproduce the characteristics of observed $\gamma$-ray flares of FSRQs (i.e. 
average flux level and minimum variability time scale). Moreover, the power spectral densities of the artificial and observed light curves are compatible within errors.

The model-predicted variability depends strongly on the orientation of the reconnection layer with respect to the blazar jet axis and to the observer. For optimal orientations, the theoretical light curves exhibit fast and powerful flares powered mostly by small and fast plasmoids. These sharp, spike-like outbursts of emission appear as excesses atop a more slowly evolving envelope produced by the cumulative emission of medium-sized plasmoids. These outbursts can easily explain minute-scale variability in FSRQs and are a clear-cut prediction of our reconnection model. A systematic search of minute-scale flares on sub-orbital time scales could serve as a test for magnetic reconnection events in blazar jets.

From a theoretical perspective, it is desirable to compute the emission produced from multiple reconnection layers formed at different distances within the jet. Extension of our radiative transfer calculations to times after plasmoids exit from the layer, where they may undergo adiabatic expansion, would be necessary for computing a likely delayed radio signal following fast $\gamma$-ray flares from reconnection.

Acknowledgements. M. M. acknowledges support from the European Research Council (ERC) under the European Union's Horizon 2020 research and innovation program Grant agreement No. 948689 (AxionDM). The Fermi-LAT Collaboration acknowledges support for LAT development, operation and data analysis from NASA and DOE (United States), CEA/Irfu and IN2P3/CNRS (France), ASI and INFN (Italy), MEXT, KEK, and JAXA (Japan), and the K.A. Wallenberg Foundation, the Swedish Research Council and the National Space Board (Sweden). Science analysis support in the operations phase from INAF (Italy) and CNES (France) is also gratefully acknowledged. This work performed in part under DOE Contract DE-AC02-76SF00515.

\section{References}

[1] M. Böttcher, Galaxies 7 (2019) 20, [1901.04178].

[2] C. M. Urry and P. Padovani, PASP 107 (1995) 803, [astro-ph/9506063].

[3] P. Padovani, D. M. Alexander, R. J. Assef et al., A\&A Rev. 25 (2017) 2, [1707.07134].

[4] W. B. Atwood, A. A. Abdo, M. Ackermann et al., ApJ 697 (2009) 1071-1102, [0902 . 1089].

[5] M. Meyer, J. D. Scargle and R. D. Blandford, ApJ 877 (2019) 39, [1902 . 02291].

[6] D. Giannios, D. A. Uzdensky and M. C. Begelman, MNRAS 402 (2010) 1649-1656, [0907.5005].

[7] K. Nalewajko, D. Giannios, M. C. Begelman et al., MNRAS 413 (2011) 333-346, [1007.3994].

[8] M. V. Barkov, F. A. Aharonian, S. V. Bogovalov et al., ApJ 749 (2012) 119, [1012 . 1787].

[9] R. Narayan and T. Piran, MNRAS 420 (2012) 604-612, [1107 . 5812].

[10] T. Hovatta, E. Valtaoja, M. Tornikoski et al., A\&A 494 (2009) 527-537, [0811.4278].

[11] J. D. Finke, ApJ 870 (2019) 28, [1811. 04999].

[12] M. C. Begelman, A. C. Fabian and M. J. Rees, MNRAS 384 (2008) L19-L23, [0709. 0540].

[13] M. Ackermann, R. Anantua, K. Asano et al., ApJ 824 (2016) L20, [1605 . 05324]. 
[14] N. F. Loureiro, A. A. Schekochihin and S. C. Cowley, Physics of Plasmas 14 (2007) 100703-100703, [astro-ph/0703631].

[15] D. A. Uzdensky, N. F. Loureiro and A. A. Schekochihin, Phys. Rev. Lett. 105 (2010) 235002, [1008.3330].

[16] Y. E. Lyubarsky, MNRAS 358 (2005) 113-119, [astro-ph/0501392].

[17] L. Sironi, D. Giannios and M. Petropoulou, MNRAS 462 (2016) 48-74, [1605. 02071].

[18] D. Giannios, MNRAS 431 (2013) 355-363, [1211.0296].

[19] M. Petropoulou, D. Giannios and L. Sironi, MNRAS 462 (2016) 3325-3343, [1606. 07447].

[20] I. M. Christie, M. Petropoulou, L. Sironi et al., MNRAS 482 (2019) 65-82, [1807.08041].

[21] M. Meyer, M. Petropoulou and I. M. Christie, ApJ 912 (2021) 40, [2012 . 09944].

[22] M. Wood, R. Caputo, E. Charles et al., in 35th International Cosmic Ray Conference (ICRC2017), vol. 301 of International Cosmic Ray Conference, p. 824, 2017, 1707.09551.

[23] D. Emmanoulopoulos, I. M. McHardy and I. E. Papadakis, MNRAS 433 (2013) 907-927, [1305.0304].

[24] W. Max-Moerbeck, J. L. Richards, T. Hovatta et al., MNRAS 445 (2014) 437-459, [1408.6265]. 